\title{
A Complex Fistula-in-Ano Presenting As A Sinus Right Side of Lower Back
}

\author{
Manish Kumar Gupta ${ }^{1}$, Anand Kumar Vijay ${ }^{2}, \mathrm{~K}_{\mathrm{M}} \mathrm{Garg}^{3}$. \\ ${ }^{l}$ Asst Professor, ${ }^{2}$ senior Resident \\ ${ }^{3}$ Professor \& Hod, Dept Of General Surgery, Jnu Imsrc, Jaipur.
}

\begin{abstract}
Anal fistula is among the most common illnesses affecting man. The majority of fistulas unrelated to specific causes (eg. Tuberculosis, Crohn's disease) result from infection (abscess) in anal glands extending from the inter sphincteric plane to various anorectal spaces. Complex variety is encountered in very few patients, which required special treatment for its cure. Extra sphincteric fistulas occur in only 5\% of total fistula-in-ano patients. We would like to report an unusual case of fistula-in-ano, where its tract reached to the right side of lower back region.
\end{abstract}

Keywords: complex, fistula-in-ano, trans-sphincteric.

\section{Introduction}

Anorectal abscess and fistula are quite commonly encountered diseases. Both of these are the acute and chronic manifestations of the same entity. While abscess are thought to begin as an infection in the anal glands, their spreading into adjacent spaces result in a fistula formation ${ }^{1}$. Many times this spread occurs in a complex pattern which is difficult to map and treat.

The accurate diagnosis and mapping of perianal fistulas has long been a challenge to surgeons word wide. Most perianal abscesses originate from an infected anal gland. Obstruction of these anal glands leads to stasis, bacterial overgrowth, and ultimately abscesses that are located in the intersphincteric groove ${ }^{2}$. These abscesses have several routes of egress, the most common of which are downward extension to the anoderm or across the external sphincter into the ischio-rectal fossa. Less common routes of spread are to the supra-levator space or in the submucosal plane.

When the abscess is drained, either surgically or spontaneously, persistence of the septic foci and epithelialization of the draining tract may occur and lead to a chronic fistula-in-ano. About $60 \%$ of the abscesses do result in to such a fistula formation ${ }^{3}$. In the presented case there was an extrasphincteric fistulous tract which extended superiorly, traversed the right ischiorectal fossa, breached the levator ani plate and presented as a chronic abscess in the subcutaneous region of the right side of lower back.

\section{Case Report}

A 47 year old male patient admitted with external sinus over medical side of right lower back region of 2 year duration, started initially as a subcutaneous abscess which was incised .This sinus opening is discharging pus off and on since then( fig.1). Specific history of recurrent discharge of pus from anus region was also present. On per rectal examination an internal fistulous opening (of about $3 \mathrm{~mm}$ size) was detected in midline posteriorly about $1 \mathrm{~cm}$ from anal verge. Patient underwent sinogram which showed contrast tracking down from external sinus tract communicating to anal canal (fig.2), simultaneously CT sinogram was performed revealing complex fistula in ano, extending from cutaneous opening in right side of lower back communicating with anal canal coursing through right paraspinal muscle, right gluteus maximus muscle, ischiorectal fossa and right levator ani muscle with few ramifications at the origin.

After bowel preparation the patient was taken to the operation room. Under general anesthesia the patient was placed in jack knife position and excision of upper part of track was done (fig.3, fig.4). On further exploration, the sinus tract was going on medial side of sacrum into pelvic cavity.

At this stage, patient position changed to lithotomy and through internal opening the track was scrapped thoroughly (cored out). Both the wounds were left open and the tract and wound were packed.

The postoperative period was largely uneventhful and the patient was discharged with advice to dress the external sinus wound site daily with povidone iodine solution after hot hip bath. There was complete healing in about 4 week. At last one year follow up visit about a month back patient was perfectly well.

A histopathological examination of the specimen obtained showed features consistent with chronic inflammation.In the follow up, a complete resolution of the fistulous tract was seen. The recovery of the patient was satisfactory. 


\section{Discussion}

A fistula-in-ano is a grannulating tract between the ano-rectum and the perineal region or perineum. A typical fistula usually consists of a tract with a primary (internal) opening and a secondary (external) opening ${ }^{1}$. The common presentation is mainly perianal suppuration and discharging sinuses. History of previous surgery and/or trauma should be ruled out initially. Also important is history of tuberculosis and any other special diseases like Crohn's disease, HIV infection etc. Digital examination usually confirms the diagnosis of lowlevel fistula in ano. Procto-sigmoidoscopy is needed to confirm high-level fistula in ano (above the dentate line). Goodsall's rule is useful for anticipating the anatomy of a simple fistula. It is of little help in defining the anatomy of complex fistulas.

Fistulography is the most easily available and reliable investigation of choice. Endo-anal ultrasound is a useful investigation in complex fistulas. CT scan is useful in extrasphincteric and retroperitoneal fistulas. MRI is emerging as investigation of choice in complex and recurrent fistulas.

When dealing with a fistula-in-ano, proper assessment and mapping of the tract is very important before going in for a definitive surgery. The aim of the fistula treatment is to remove the entire pathology from its root and to preserve anal continence ${ }^{8}$. The most commonly used surgical techniques for the treatment of branching fistulas include a two-stage excision of fistula and a one-stage approach ${ }^{5}$.

The two-stage fistulectomy involves, in the first stage, the excision of the extra-sphincteric portion of fistula tract along with the adjacent secondary tracts as well. The second stage involves an incision of the transsphincteric portion or an incision with a subsequent reconstruction of the transected part of the external sphincter. In some cases the surgery involves several stages. This most often applies to fistulas with multiple tracts or a situation when there are uncertainties about their presence. One-stage procedures for anal fistula include fistulotomy, fistulectomy, seton, fibrin glue, advancement flap, and coring and anal fistula plug.

In cases of complex recurring anal fistulae the use of imaging techniques should be considered ${ }^{6}$. Magnetic resonance imaging (MRI) may also be helpful, especially in cases of supra-sphincteric and extrasphincteric fistulae. The tract of the fistula and its relationship to the sphincter muscle can be investigated by probing and/or using dye intra-operatively with the patient under anesthesia ${ }^{7}$. Complex fistula has secondary tracks or extensions apart from curved primary track which crosses external anal sphincter (EAS) that can make eradication of sepsis very difficult. Fistulotomy is not recommended because of fear of cutting significant amount of EAS.

In the presented case, a CT sinogram delineated an extra-sphincteric type of fistula extending from cutaneous opening in right side of lower back communicating with anal canal coursing through right paraspinal muscle, right gluteus maximus muscle, ischiorectal fossa and right levator ani muscle. In this patient excision of upper part of track was done (fig.3), further the track was going on medial side of sacrum into pelvic cavity(fig.4), so patient position changed from jack knife to lithotomy and thorough scrapping of tract was done through internal opening which was situated in posterior midline of anal canal $1 \mathrm{~cm}$ from anal verge.

Hence, treatment of complex fistulas poses a major challenge for surgeons. The following elements of surgical technique should be taken into consideration (a) Identification of internal opening (b) Identification with subsequent staining with methylene blue of the main canal and secondary tracts (c) Excision of main fistula canal followed by removal of all secondary tracts, if possible. If the canals are located high and cannot be excised under direct vision, they should be opened and carefully curetted ${ }^{5}$.

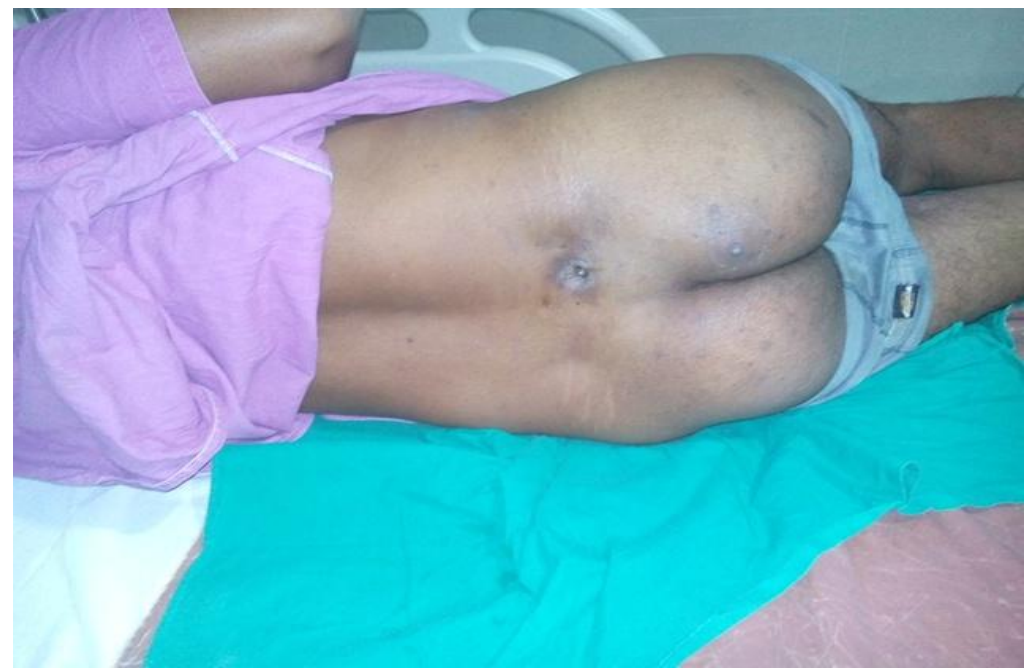

Fig 1 : showing external opening over right side of lower back region 


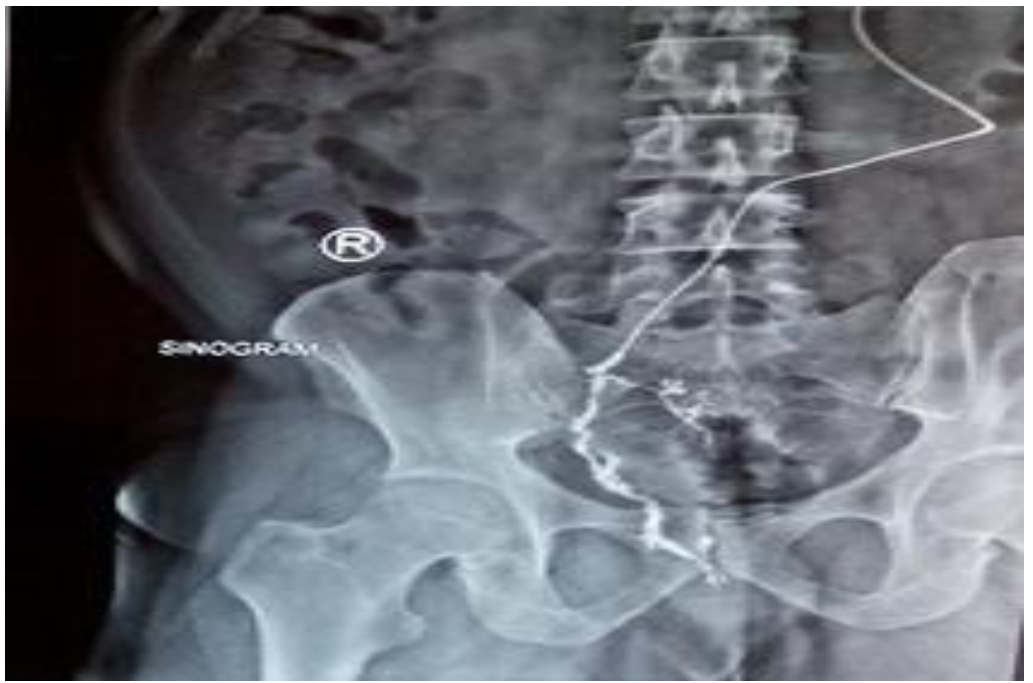

Fig 2 : Sinogram showing contrast trackling down from external sinus tract

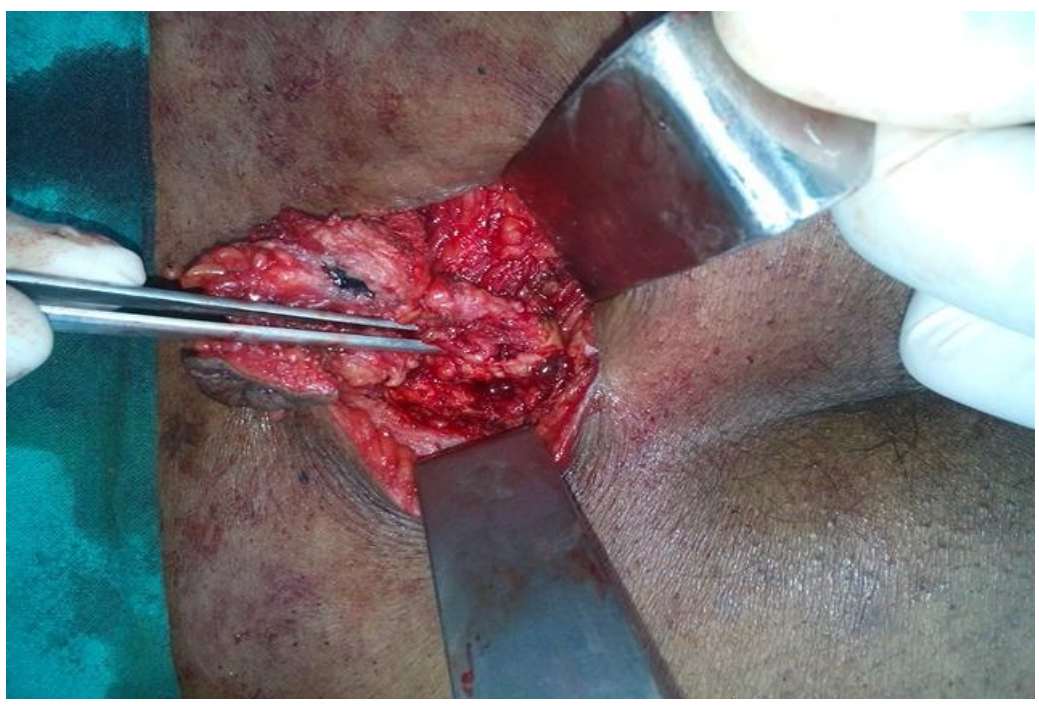

Fig.3 : Excision of upper parte of track in jack knife Position E

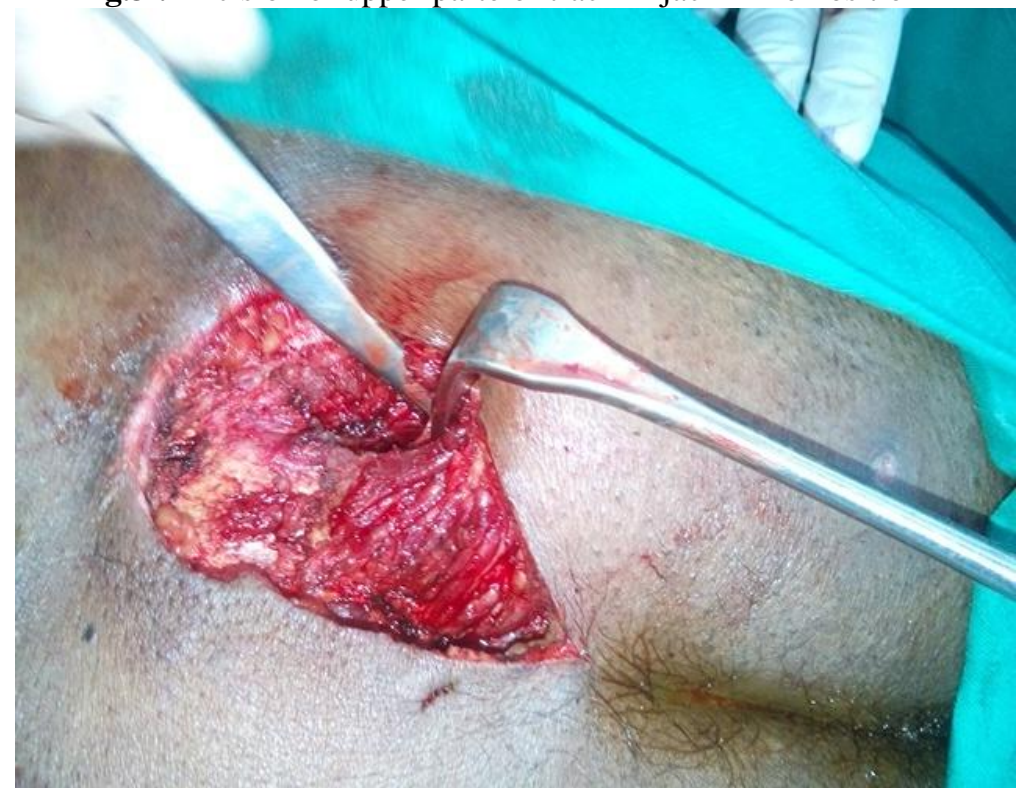

Fig.4 : After excision of upper part of track and scrapping from above 


\section{References}

[1]. Srivastava K N and Amit Agarwal . A complex fistula-in-ano presenting as a soft tissue tumor.

a. $\quad$ Int.Surg Case Rep. 2014; 5 (6) : 298-301

[2]. Parks A G. Pathogenesis and treatment of fistula-in-ano. B M J $1961 ; 1$ : 463-469[ Pub Med ]

[3]. Robinson A M ,Jr, DeNobile J W. Anorectal abscess and fistula-in-ano. J Natl Med Assoc. 1988; (11) : 1209-1213 [ Pub Med ]

[4]. Belekar D, Dewoolkar V, Desai A, Anam J, Parab M. An unusual case of complex transsphincteric fistula-in-ano. ISPUB.Com/ The Internet Journal of Surgery vol 19, no2/11778, 2017

[5]. Anna Wiaczek, Malgorata Kolodziejczak, Iwona Sudol-Szopinska. Diagnosis and surgical treatment of complex anorectal fistulas. Nowa Medycyna 1/ 2016, s. 29-37.

[6]. Zbar AP, Armitage NC. Complex perirectal sepsis : clinical classification and imaging. Tech Coloproctol. 2006; 10 : 83-93.

[7]. Gonzalez Ruiz C, Kaiser AM, Vukasin P, et al. Intraoperative physical diagnosis in the management of anal fistula. Am Surg. 2006 ; $72: 11-15$

[8]. Imam Uddin, Hina Abdul Qayoom Khan, M. Shamim Qureshi. Complex fistula-in-ano : surgical management and outcome. Journal of Surgery Pakistan (International) 19 (4) oct-dec 2014, 132-135 